\title{
Use of Symmetry for Residual Stress Determination
}

\author{
Robert Charles Wimpory ${ }^{1, a}{ }^{*}$ and Michael Hofmann ${ }^{2, b}$ \\ ${ }^{1}$ Helmholtz Centre Berlin for Materials and Energy, Hahn-Meitner-Platz 1, 14109 Berlin, \\ Germany \\ ${ }^{2}$ Heinz Maier-Leibnitz Zentrum (MLZ), Technische Universität München, Lichtenbergstr. 1, \\ 85748 Garching, Germany \\ a robert.wimpory@helmholtz-berlin.de, ${ }^{\mathrm{b}}$ Michael.Hofmann@frm2.tum.de
}

Keywords: Surface Effect, Absorption Effect, Neutron Diffraction, Aberrations, Round Robin, Standardization, Grain Size Effects, Plane Stress Calculations, Nickel Alloy

\begin{abstract}
Instrumental and certain sample characteristics can affect the detected Bragg peak shifts which are not related to the strain being measured. Three major effects can influence the measurement: the surface effect, where the instrumental gauge volume (IGV) is not fully immersed at a surface or interface, the grain size effect where there is random positioning of large grains in the sample within the gauge volume and the relative shift in position of the centre of gravity of measurement due to absorption of neutrons. All of these effects can be reduced/eliminated by making pairs of neutron diffraction measurements 180 degrees to each other at the same location. Results are presented from a round robin benchmark weldment, denoted TG6, from the European Network on Neutron Techniques Standardization for Structural Integrity (NeT). This is made from a nickel alloy which has large grains and strains and has a high neutron attenuation coefficient.
\end{abstract}

\section{Introduction}

The surface effect, where the instrumental gauge volume (IGV) is not fully immersed at a surface or interface, normally results in a detected shift of the Bragg-peak and is not related to strain. Webster et al [1] noticed that the instrumental surface effect is essentially symmetrical. This aberration is influenced by a number of parameters, such as the bending radius when using a perfectly bent monochromator [2, 3] and is therefore instrument specific. Figure 1 shows the surface effect when measuring with a $2 \times 2 \times 10 \mathrm{~mm}^{3}$ gauge volume on E3 at the HZB [4] (where $10 \mathrm{~mm}$ is the gauge volume height) on a nominally strain-free $10 \mathrm{~mm}$ thick ferritic steel sample. Making pairs of measurements $180^{\circ}$ to each other show the symmetrical nature of the surface effect. The bending radius of the Si [400] monochromator is set fortuitously so the surface effect is almost canceled out for the out-of-plane normal direction for this particular 2theta scattering angle. Averaging the pairs of measurements for each corresponding position results in values that are consistent from the surface to the inside of the specimen (see table 1). Holden et al [5] found that for large-grained samples making pairs of neutron diffraction measurements $180^{\circ}$ to each other is a way of minimizing the scatter coming from the random positioning of grains within the gauge volume. Also the relative shift in position of the centre of gravity of measurement due to absorption of neutrons [6] can be cancelled out using this $180^{\circ}$ technique. The following results presented are from a round robin benchmark weldment, denoted TG6, from the European Network on Neutron Techniques Standardization for Structural Integrity (NeT). This is made from a nickel alloy which has large grains, large strains and a high neutron attenuation coefficient, making it ideal for testing out the ' $180^{\circ}$ ' technique. 


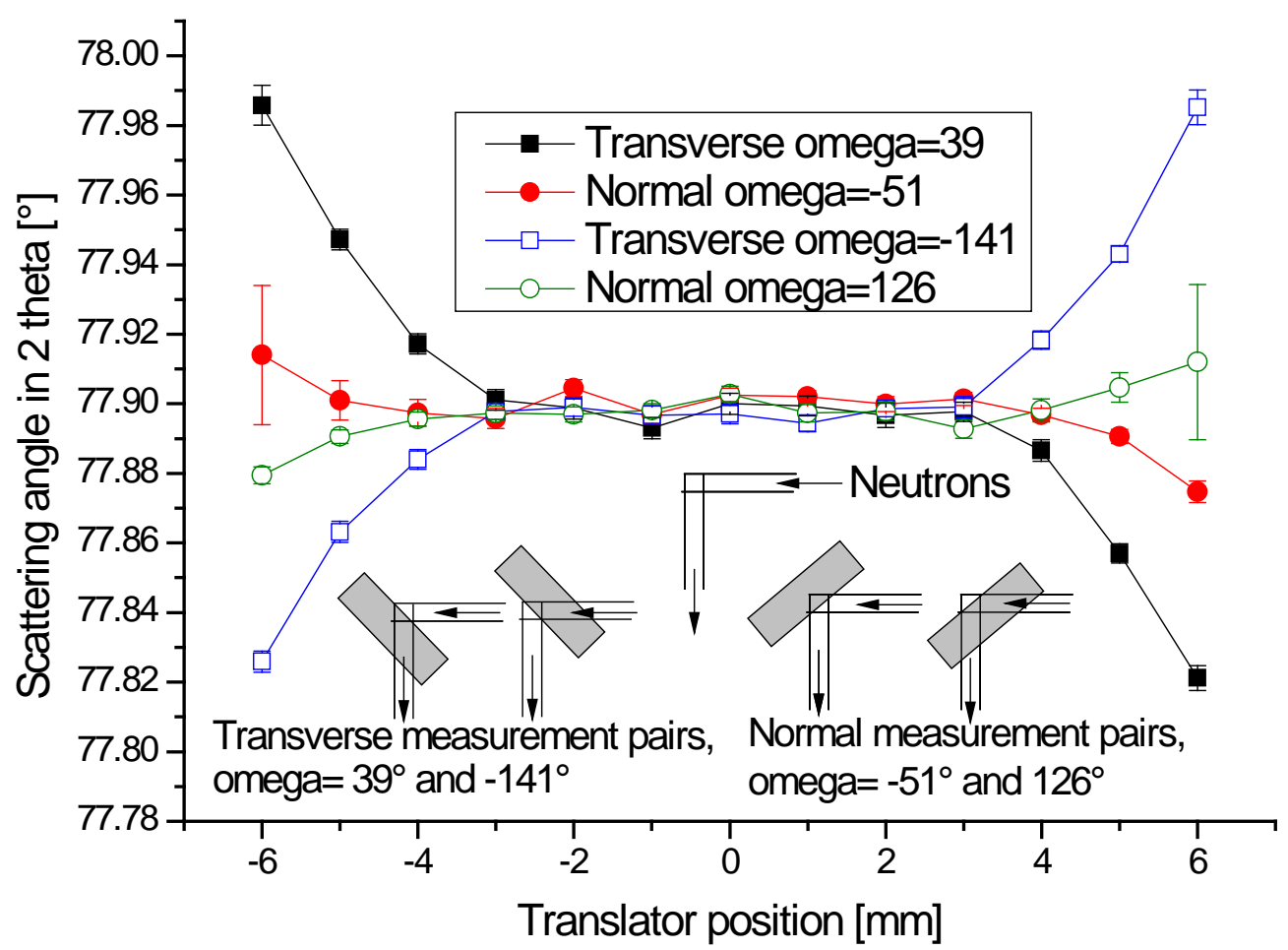

Figure 1. The surface effect of in-plane (transverse) and out of plane (normal) direction on E3.

Table 1. Measurement on a nominally strain-free $10 \mathrm{~mm}$ thick ferritic steel sample.

\begin{tabular}{|c|c|c|c|c|c|c|c|}
\hline $\begin{array}{c}\text { Translator } \\
\text { position } \\
\text { [mm] }\end{array}$ & $\begin{array}{l}\text { Actual } \\
\text { position } \\
\text { [mm] }\end{array}$ & $\begin{array}{c}\text { Transverse } \\
\omega=39 \\
\left.{ }^{\circ}\right]\end{array}$ & $\begin{array}{c}\text { Normal } \omega \\
=-51 \\
{\left[^{\circ}\right]}\end{array}$ & $\begin{array}{c}\text { Transverse } \\
\omega=-141 \\
{\left[^{\circ}\right]}\end{array}$ & $\begin{array}{c}\text { Normal } \\
\omega=126 \\
{\left[^{\circ}\right]}\end{array}$ & $\begin{array}{c}\text { Transverse } \\
\text { Average } \\
{\left[{ }^{\circ}\right]}\end{array}$ & $\begin{array}{c}\text { Normal } \\
\text { Average } \\
{\left[{ }^{\circ}\right]}\end{array}$ \\
\hline-6.00 & -4.86 & 77.9858 & 77.9140 & 77.8259 & 77.8794 & 77.9059 & 77.8967 \\
\hline-5.00 & -4.51 & 77.9472 & 77.9010 & 77.8632 & 77.8906 & 77.9052 & 77.8958 \\
\hline-4.00 & -3.88 & 77.9172 & 77.8974 & 77.8840 & 77.8956 & 77.9006 & 77.8965 \\
\hline-3.00 & -3.00 & 77.9011 & 77.8958 & 77.8978 & 77.8974 & 77.8995 & 77.8966 \\
\hline-2.00 & -2.00 & 77.8988 & 77.9045 & 77.8990 & 77.8970 & 77.8989 & 77.9008 \\
\hline-1.00 & -1.00 & 77.8930 & 77.8969 & 77.8967 & 77.8981 & 77.8949 & 77.8975 \\
\hline 0.00 & 0.00 & 77.9000 & 77.9025 & 77.8971 & 77.9028 & 77.8986 & 77.9027 \\
\hline 1.00 & 1.00 & 77.8994 & 77.9021 & 77.8944 & 77.8973 & 77.8969 & 77.8997 \\
\hline 2.00 & 2.00 & 77.8967 & 77.8999 & 77.8986 & 77.8978 & 77.8977 & 77.8989 \\
\hline 3.00 & 3.00 & 77.8977 & 77.9014 & 77.8991 & 77.8928 & 77.8984 & 77.8971 \\
\hline 4.00 & 3.88 & 77.8866 & 77.8968 & 77.9183 & 77.8982 & 77.9025 & 77.8975 \\
\hline 5.00 & 4.51 & 77.8570 & 77.8906 & 77.9430 & 77.9047 & 77.9000 & 77.8977 \\
\hline 6.00 & 4.86 & 77.8212 & 77.8747 & 77.9852 & 77.9120 & 77.9032 & 77.8934 \\
\hline \multirow{2}{*}{\multicolumn{6}{|c|}{$\begin{array}{l}\text { Average of averages } \\
\text { Standard deviation }\end{array}$}} & 77.9002 & 77.8977 \\
\hline & & & & & & 0.0032 & 0.0023 \\
\hline
\end{tabular}




\section{Results}

The TG6 test component comprises a $200 \mathrm{~mm} \times 150 \mathrm{~mm} \times 12 \mathrm{~mm}$ rectangular base plate made from Inconel 600 with three passes of Alloy 82 weld metal deposited in a slot of length $76 \mathrm{~mm}$. This not only has large grain issues in the weld region but a large interplanar spacing variation due to high strain gradients and a change in material composition. Each position was measured on E3 in steps of 1 degree, -3,-2-1,0,2,3 about the scattering vector and the value was then averaged. This was to reduce grain size effects [7]. Figure 2 shows the measurement of longitudinal direction (which is made in transmission geometry) along the central line of the TG6 specimen (denoted the BD line) using a $2 \times 2 \times 2 \mathrm{~mm}^{3}$ gauge volume. This is the third measurement of the BD line on E3 (July 2017). The bulge at the back of the weld was set to $y=12 \mathrm{~mm}$. The back plate surfaces immediately to the sides of the bulge were $y=11.5 \mathrm{~mm}$. The total length of the BD line is $14.59 \mathrm{~mm}$. The reference pin (denoted pin 3) is actually $14.36 \mathrm{~mm}$, one surface however should correspond to the back face of the weld, i.e. the surface of the bulge at $\mathrm{y}=12 \mathrm{~mm}$. The bottom of the weld is at about $\mathrm{y}=5 \mathrm{~mm}$, where the parent material is from $\mathrm{y}=5$ to $12 \mathrm{~mm}$.

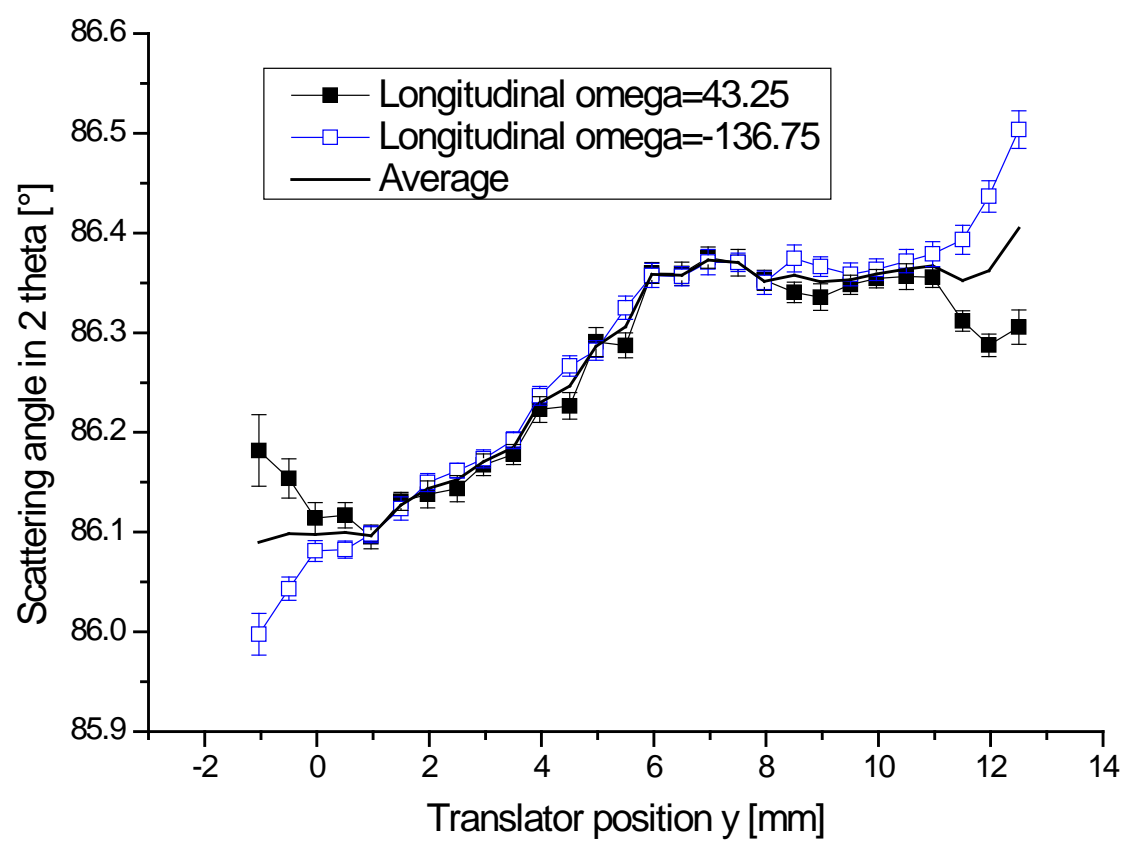

Figure 2. Measurement of the longitudinal direction along the BD line in the TG6 specimen.

The figure shows that there is indeed the surface effect as the gauge volume exits the surface. The coincidence of the lines where the gauge volume is fully submersed proves the good alignment of the primary slit over the centre of rotation of the diffractometer's omega table. The measurement was made in transmission geometry. Conversely the normal direction was measured in reflection geometry. This is susceptible to an absorption effect [6] which can be clearly seen in figure 3 . This is because the centre of gravity of the gauge volume in terms of scattered neutrons in refection geometry is at slightly different position to the geometrical centre of the gauge volume. Measurement however is difficult on E3 over the complete BD line from one side as the neutron absorption of the nickel alloy is very high. Taking an average of the 
values where there are two values gives the correct value for the position [6]. It is advised that the region where one can do overlapping measurements is measured first so one can find the absorption shift, so one can work out the translator positions that correspond to the intended measurement positions.

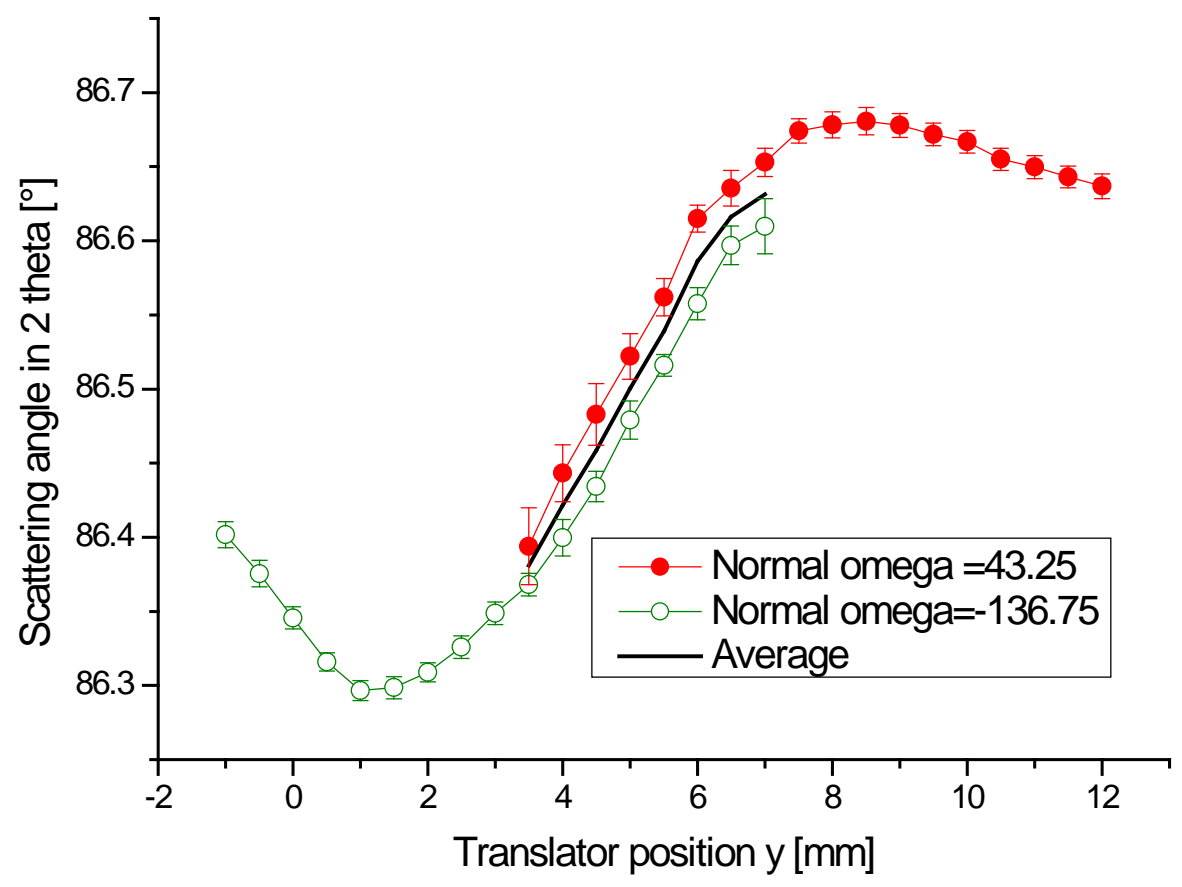

Figure 3. Measurement of normal direction along the BD line in the TG6 specimen on E3.

A $5 \mathrm{~mm}$ slice of the weld was also available which turned out to be better for relative positioning of corresponding reference values for the welded plate compared to the pin (see figure 4). The stress is much smaller in the slice compared to the plate. Two plane stress calculations were made in the slice (assuming the longitudinal stress and normal stress directions were zero respectively) and these agree with each other well. For a comparison, the reference value needed to set the normal direction $=0 \mathrm{MPa}$ in the welded plate is also depicted and this agrees with the slice positioning. One can fit the distribution with a Sigmoidal function and the centre positon of the three values obtain agrees to within $4.87 \pm 0.06 \mathrm{~mm}$. There is a slight offset with respect with the reference pin which was $4.56 \pm 0.04 \mathrm{~mm}$. This corresponds to the difference in length of the pin $(14.36 \mathrm{~mm})$ and the actual length of the BD line $(14.59 \mathrm{~mm})$ to within experimental uncertainty.

Final stress calculations agree mostly very well with measurements made on Stress-Spec [8] at the FRMII (see figure 5). The normal strain was also made using the ' $180^{\circ}$ ' technique but one was able to measure along the whole BD line due to the higher flux at FRMII and so the average values were made across the whole of the BD line. Agreement of the normal stress in the weld near the surface is not so good; this may be due to grain size issues that were not canceled out due to normal strain not being completely measured from both sides on E3. The best agreement is in the parent region where the grain size is smaller. The normal stress at the surface is $0 \mathrm{MPa}$ 
as expected. A value of near $0 \mathrm{MPa}$ was also obtained on the Stress-Spec measurement in the weld region.

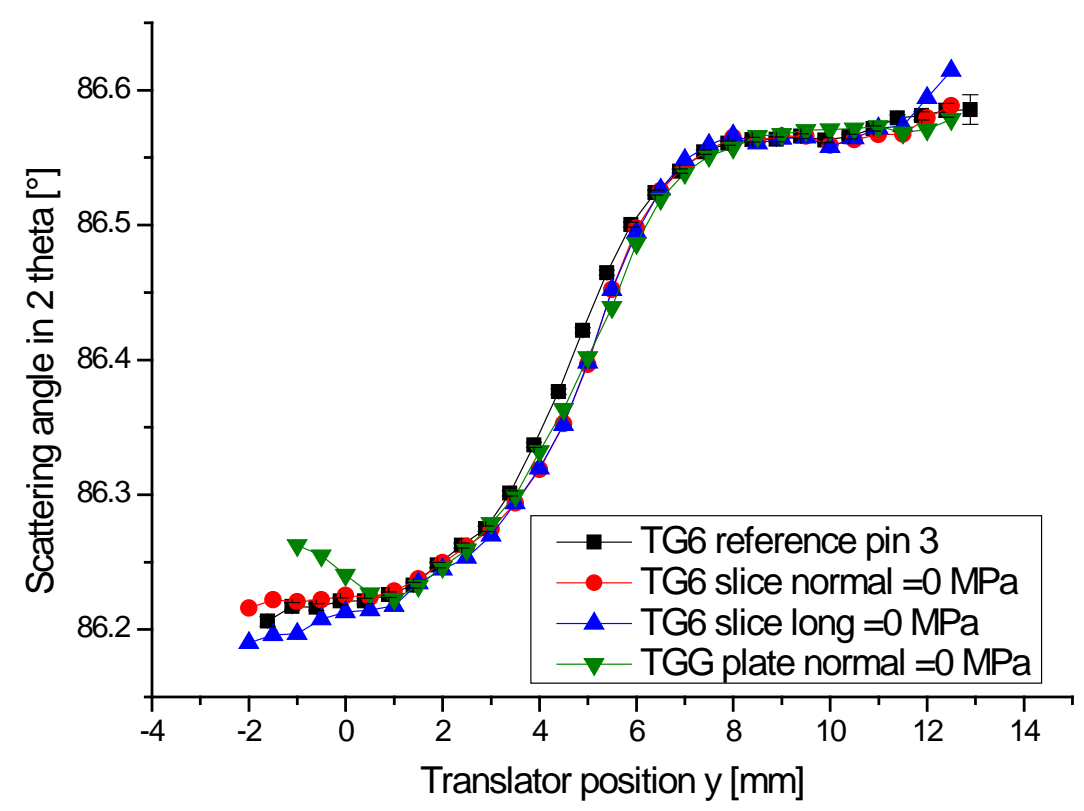

Figure 4. Comparison of reference values obtained from the TG6 pin, slice and plate.

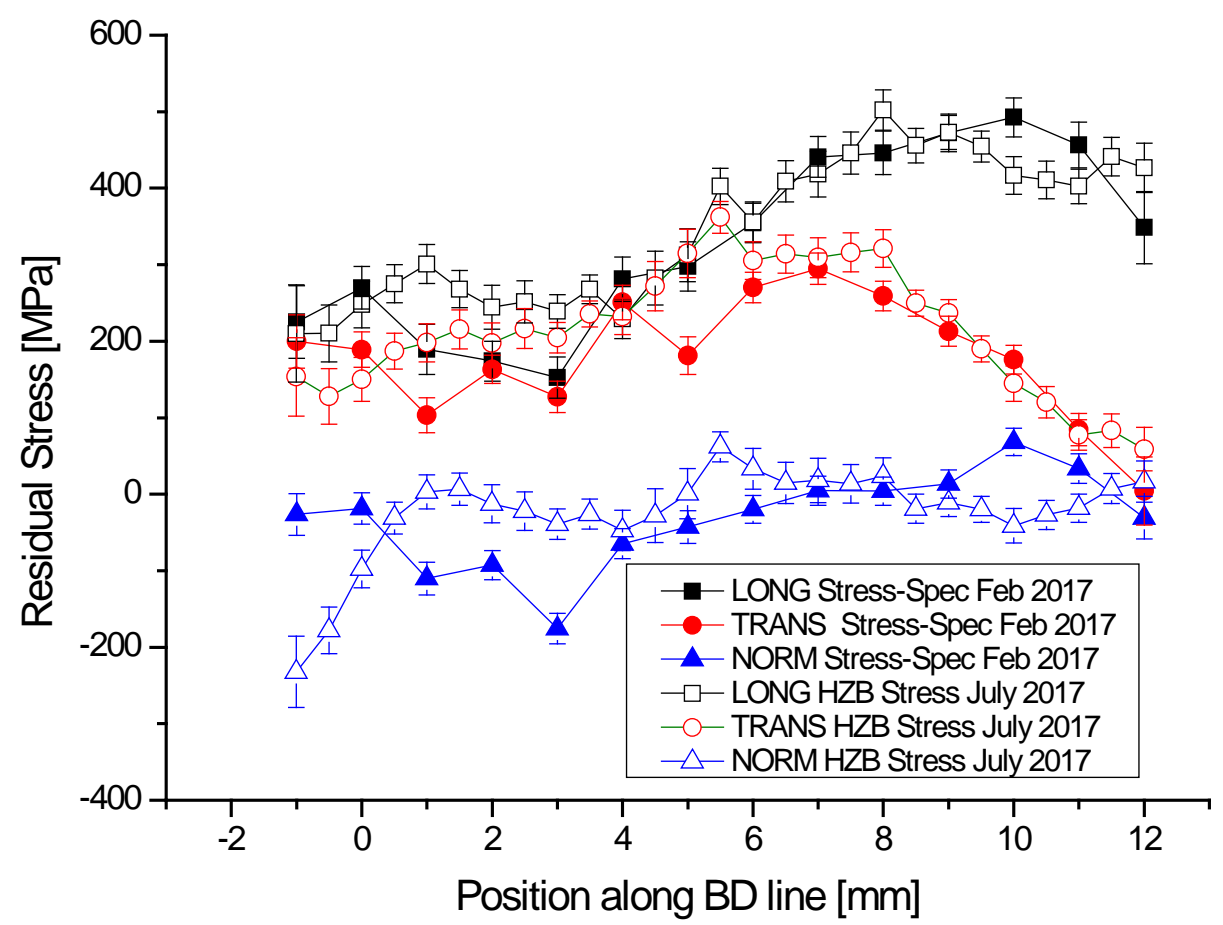

Figure 5. Comparison of stress from Stress-Spec and E3 at the HZB along the BD plate line. 


\section{Summary}

The Net TG6 round robin specimen presented itself to be a challenge when determining the strain and stress using neutrons. Measuring the normal strain can be difficult when there is a large neutron path length and getting a complete overlap of measurements is challenging when using the $180^{\circ}$ technique. Agreement of the normal stress in the two measurements presented in the weld near the surface is not so good; this may be due to grain size issues that were not canceled out due to normal strain not being completely measured from both sides on E3. The best agreement is in the parent region where the grain size is smaller. The normal stress at the surface is $0 \mathrm{MPa}$ as expected. A value of near $0 \mathrm{MPa}$ was also obtained on the Stress-Spec measurement in the weld region. In general using the $180^{\circ}$ technique is useful for measuring near surfaces to get a more accurate value of strain. This technique also cancels out the absorption effect [6] and also can be used for grain size effect mitigation [5]. The sample has a large interplanar spacing variation due to high strain gradients and a change in material composition. The gradients due to only the material composition can be seen in figure 4. A Sigmoidal function can be used to fit these reference distributions to obtain a better positioning to obtain accurate strain values in the welded plate.

\section{References}

[1] Webster, P.J., Mills, G. , Wang, X.D. , Kang, W.P. , Holden, T.M. 'Impediments to Efficient Through-Surface Strain Scanning’, Journal of Neutron Research, vol. 3, no. 4, pp. 223-240, 1996. https://doi.org/10.1080/10238169608200197

[2] M. Vrána and P. Mikula, "Suppression of Surface Effect by Using Bent-Perfect-Crystal Monochromator in Residual Strain Scanning", Materials Science Forum, Vols. 490-491, pp. 234238, 2005. https://doi.org/10.4028/www.scientific.net/MSF.490-491.234

[3] Saroun, J., Kornmeier, J. R., Hofmann, M., Mikula, P. \& Vrána, M. (2013). J. Appl. Cryst. 46, 628-638. https://doi.org/10.1107/S0021889813008194

[4] Wimpory, R. C., Mikula, P., Šaroun, J., Poeste, T., Li, J., Hofmann, M. and Schneider, R. Neutron News, 19, (2008) , 16 - 19. https://doi.org/10.1080/10448630701831995

[5] Holden, T. M., Traore, Y., James, J., Kelleher, J. \& Bouchard, P. J. (2015). J. Appl. Cryst. 48, 582-584. https://doi.org/10.1107/S1600576715002757

[6] "Precise measurement of steep residual strain gradients using neutron diffraction in strongly absorbing materials with chemical compositional gradients", Robert C. Wimpory, Michael Hofmann, Vasileios Akrivos, Mike C Smith, Thilo Pirling and Carsten Ohms., Accepted for publication in Materials Performance and Characterization 2018

[7] Robert Charles Wimpory, René V. Martins, Michael Hofmann, Joana Rebelo Kornmeier, Shanmukha Moturu, Carsten Ohms, International Journal of Pressure Vessels and Piping, 2017, ISSN 0308-0161. https://doi.org/10.1016/j.ijpvp.2017.09.002

[8] Hofmann, M., Schneider, R., Seidl, G.A, Kornmeier, J, Wimpory, R., Garbe, U. und Brokmeier, H.G., Physica B, 2006, 385 - 368, 1035 - 1037 